\title{
Сиделов Д.И.
}

\section{О необходимости применения параксиального приближения при решении олимпиадных задач геометрической оптики}

\author{
Sidelov D.I. \\ The need for the use of the paraxial approximation in \\ solving olympiad problems of geometrical optics
}

В статье рассматривается цепочка олимпиадных физических задач геометрической оптики, при решении которых обязательно использование параксиального приближения

Ключевые слова: олимпиадные задачи, геометрическая оптика, параксиальное приближение

\section{Сиделов Дмитрий Ильич}

Кандидат физико-математических наук, доцент Оренбургский государственный педагогический университет

г. Оренбург, ул. Советская, 19
The article discusses the chain olympiad physics problems of geometrical optics, the solution of which required the use of the paraxial approximation

Key words: olympiad problems, geometrical optics, paraxial approximation

\section{Sidelov Dmitry Ilyich}

Candidate of Physical and Mathematical Sciences, Associate Professor Orenburg State Pedagogical University Orenburg, Sovetskaya st., 19

В геометрической оптике существует ряд задач повышенного уровня сложности, при решении которых параксиальное приближение является одним из основных способов получения ответа на вопрос задачи [1, с. 280]. При таком подходе пучки лучей света считаются узкими, мало отходящими от главной оптической оси линзы или сферического зеркала, что позволяет значительно упростить выводы формул, по которым можно рассчитать фокусные расстояния этих оптических систем.

Обсудим «плюсы» и «минусы» параксиального приближения. К числу неоспоримых преимуществ такого способа решения задач геометрической оптики следует отнести, в первую очередь, возможность замены тригонометрических функций синуса и тангенса самими значениями углов, вследствие малости последних, т.е. $\sin \alpha \approx \operatorname{tg} \alpha \approx \alpha$.

Такая замена значительно облегчает решение целого ряда задач, связанных с отражением и преломлением на границах раздела прозрачных диэлектриков. При этом сложное для решения в общем случае тригонометрическое уравнение вырождается в обычное линейное, к примеру, закон преломления В. 
Снеллиуса можно записать в параксиальном приближении следующим обра30M:

$$
\left\{\begin{array}{l}
\mathrm{n}_{1} \cdot \sin \alpha=\mathrm{n}_{2} \cdot \sin \beta \\
\lim _{\alpha \rightarrow 0} \sin \alpha=\alpha \\
\lim _{\beta \rightarrow 0} \sin \beta=\beta
\end{array} \mid \Rightarrow \mathrm{n}_{1} \cdot \alpha=\mathrm{n}_{2} \cdot \beta .\right.
$$

Те же выводы можно привести и для случаев замены тангенсов углов самими углами. Кроме того, в случае малого значения угла длины дуги и хорды, опирающиеся на этот угол, одинаковы.

Конечно, как и любое приближение, параксиальное имеет свои существенные недостатки. В реальных оптических системах необходимо получать как можно более светосильные схемы, если же пользоваться только параксиальными пучками лучей, то изображение даже хорошо освещенных предметов будет характеризоваться слабой освещенностью. Заметим, что получение параксиальных пучков лучей подразумевает использование в оптической схеме прибора диафрагмы, которая в свою очередь будет нагреваться задерживаемым светом (электромагнитными волнами). Также на малом отверстии диафрагмы будет наблюдаться явление дифракции, что приводит к дефокусировке изображения.

Все вышеперечисленные недостатки параксиального приближения относятся в большей мере именно к практической оптике и не умаляют его ценности при решении задач теоретической направленности.

В качестве примера применения параксиального приближения рассмотрим вывод формулы отражения от сферического зеркала. Выполним построение для вогнутого зеркала.

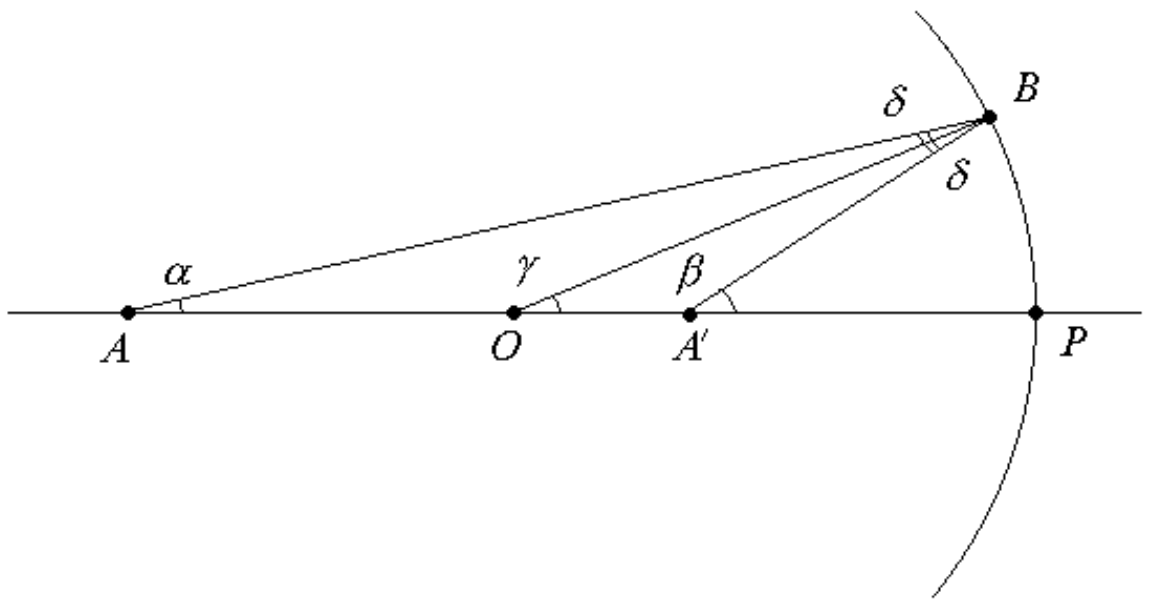

Рис. 1 Ход лучей при отражении от сферического зеркала

На рисунке изображены: предмет - точка А, изображение предмета - точка $\mathrm{A}^{\prime}$, центр сферической поверхности - O, полюс зеркала - P, В - точка отражения луча от зеркала. 
В условиях параксиального приближения можно считать, что:

$$
\mathrm{AP}=\mathrm{AB}=\mathrm{a}, \mathrm{A}^{\prime} \mathrm{P}=\mathrm{A}^{\prime} \mathrm{B}=\mathrm{b}, \mathrm{OP}=\mathrm{OB}=\mathrm{R} .
$$

Поскольку все углы в нашей схеме малые, то их можно определить по формуле длины дуги, опирающейся на угол:

$$
\alpha=\mathrm{BP} / \mathrm{a}, \beta=\mathrm{BP} / \mathrm{b}, \gamma=\mathrm{BP} / \mathrm{R} .
$$

Из теоремы о смежных углах треугольников (смежный угол равен сумме двух других углов треугольника) получаем:

$$
\left\{\begin{array}{l}
\gamma=\alpha+\delta \\
\beta=\gamma+\delta
\end{array} \Rightarrow \alpha+\beta=2 \gamma\right.
$$

Подставим в полученное выражение обозначения углов:

$$
\frac{\mathrm{BP}}{\mathrm{a}}+\frac{\mathrm{BP}}{\mathrm{b}}=2 \frac{\mathrm{BP}}{\mathrm{R}} \text {. }
$$

Сокращая $B P$, приходим к формуле сферического зеркала -

$$
\frac{1}{\mathrm{a}}+\frac{1}{\mathrm{~b}}=\frac{2}{\mathrm{R}} \text {. }
$$

Проанализируем полученное выражение. Фокус зеркала - это точка, в которой собираются при отражении лучи, шедшие параллельно главной оптической оси. Такой случай соответствует бесконечно удаленному точечному источнику, т.е. $\mathrm{a} \rightarrow \infty$, тогда $\mathrm{F}=\lim _{\mathrm{a} \rightarrow \infty} \mathrm{b}$, где $\mathrm{F}$ - фокусное расстояние зеркала.

$$
\begin{gathered}
b=\frac{R \cdot a}{2 a-R}=\frac{R}{2-R / a} \\
F=\lim _{a \rightarrow \infty} b=\lim _{a \rightarrow \infty} \frac{R}{2-R / a}=\frac{R}{2} .
\end{gathered}
$$

Покажем насколько просто можно вывести формулу для преломления на сферической границе раздела двух сред.

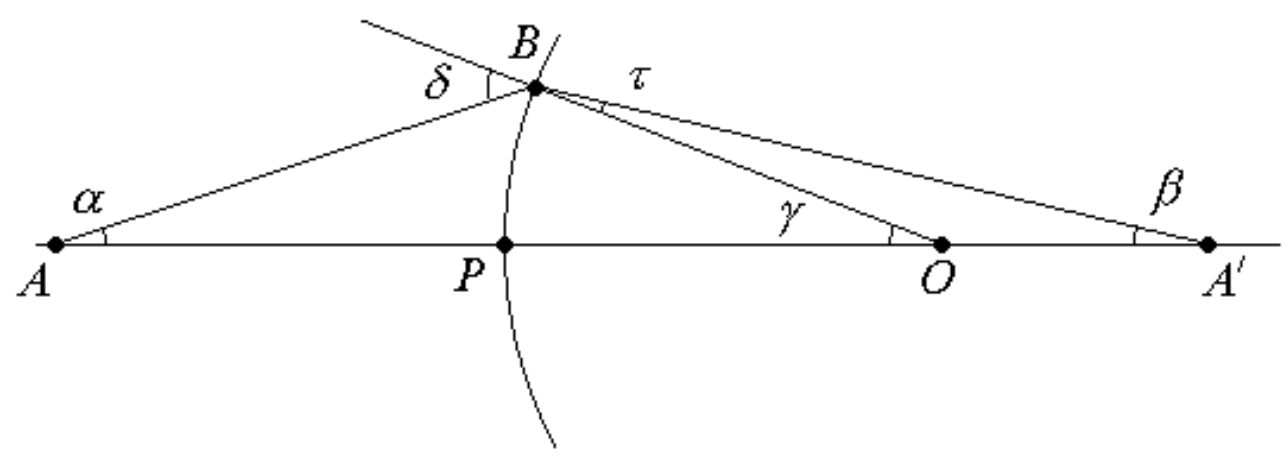

Рис. 2. Ход лучей в прочессе преломления на сферической границе раздела двух сред

Обозначения точек те же, что и в предыдущем примере. Известно, что унификация обозначений улучшает восприятие преподаваемого материала. 
В условиях параксиального приближения можно считать, что:

$$
\mathrm{AP}=\mathrm{AB}=\mathrm{a}, \mathrm{A}^{\prime} \mathrm{P}=\mathrm{A}^{\prime} \mathrm{B}=\mathrm{b}, \mathrm{OP}=\mathrm{OB}=\mathrm{R} .
$$

Обозначим углы:

$$
\alpha=\mathrm{BP} / \mathrm{a}, \beta=\mathrm{BP} / \mathrm{b}, \gamma=\mathrm{BP} / \mathrm{R} .
$$

Предположим, что относительный показатель преломления среды второй среды (изображения, точка $\mathrm{A}^{\prime}$ ) относительно первой (предмета, точка $\mathrm{A}$ ) равен $\mathrm{n}$. Тогда закон преломления с учетом малости углов падения и преломления примет вид:

$$
\delta=\tau \cdot n
$$

Из теоремы о смежных углах треугольников получаем:

$$
\left\{\begin{array} { l } 
{ \delta = \alpha + \gamma } \\
{ \gamma = \tau + \beta }
\end{array} \Rightarrow \left\{\begin{array}{c}
\tau \cdot n=\alpha+\gamma \\
\tau=\gamma-\beta
\end{array} \Rightarrow \alpha+\gamma=(\gamma-\beta) n \Rightarrow \alpha+\beta n=\gamma(n-1)\right.\right. \text {. }
$$

Подставим обозначения углов:

$$
\frac{\mathrm{BP}}{\mathrm{a}}+\frac{\mathrm{BP}}{\mathrm{b}} \mathrm{n}=\frac{\mathrm{BP}}{\mathrm{R}}(\mathrm{n}-1)
$$

Окончательное выражение получается после сокращения ВР -

$$
\frac{1}{\mathrm{a}}+\frac{\mathrm{n}}{\mathrm{b}}=\frac{\mathrm{n}-1}{\mathrm{R}} \text {. }
$$

Таким образом, мы убедились в упрощении выводов формул отражения от сферического зеркала и преломления на сферической поверхности раздела двух сред. Особенную ценность вывода представляет унификация обозначений необходимых для вывода углов и точек на схемах, выражающих ход лучей в этих случаях. Унификация обозначений крайне упрощает процесс обучения и последующего тренинга будущих участников олимпиад, поскольку порядок в обозначениях влечет за собой обязательное упорядочивание в логических схемах мышления обучаемых. Заметим также, что в наших унифицированных обозначениях отсутствуют нижние индексы, что позволяет учащимся воспринимать получаемую информацию без искажений, не отвлекаясь на анализ более мелкого, но не менее важного текста.

Рассмотрим цепочку олимпиадных задач геометрической оптики, которые можно использовать в процессе тренинга учащихся-олимпиадников. Для решения их будем использовать параксиальное приближение.

Задача 1. Со стороны основания пустотелого конуса высоты h с малым углом при вершине отрезали небольшое кольцо и поместили в параллельный пучок света, широкой частью в сторону пучка. На каком расстоянии от кольца сфокусируются отразившиеся от него лучи света [2, с. 308].

Решение:

Пусть падающий на кольцо луч отражается от него в точке М и пересекает ось конуса в точке К. Очевидно, что К - есть искомая точка фокусировки отраженных от кольца лучей. Тогда пользуясь законом отражения и рассматривая углы скольжения падающего и отраженного от зеркала лучей равные $\varphi$, можно найти по теореме о смежном угле треугольника $\angle \mathrm{LKM}=\varphi+\varphi=2 \varphi$. 


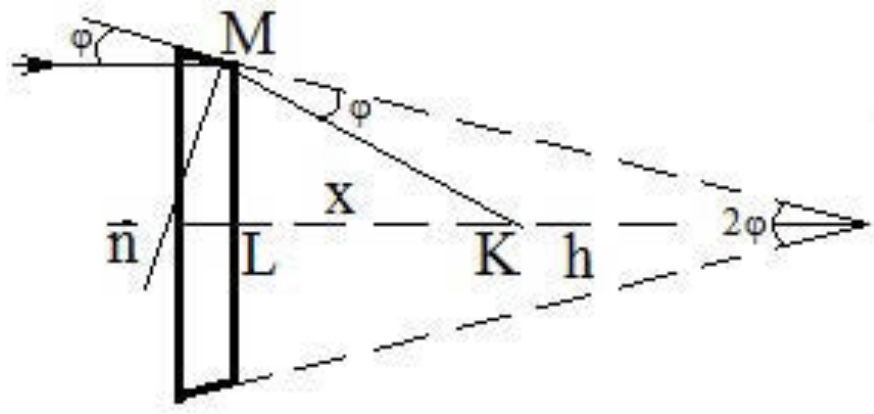

Рис. 3. Отражение от конического кольца

Найдем радиус кольца $\mathrm{ML}=\mathrm{h} \cdot \operatorname{tg} \varphi=\mathrm{x} \cdot \operatorname{tg} 2 \varphi$. Учитывая малость угла $2 \varphi \rightarrow 0$, заменим тангенсы углов их значениями, выраженными в радианах, тогда

$$
\mathrm{h} \cdot \varphi=\mathrm{x} \cdot 2 \varphi \Rightarrow \mathrm{x}=\frac{\mathrm{h}}{2} .
$$

Задача 2. Кажущаяся глубина водоема 3 м. Какова его истинная глубина? [2, с. 309]

Решение.

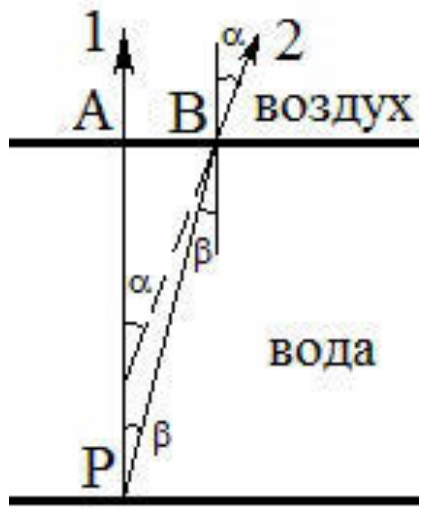

Рис. 4. Ход лучей при получении

изображения дна водоема $\beta$

Пусть точка $\mathrm{P}$ на дне водоема отражает падающий на нее солнечный свет и испускает два луча 1 и 2, которые при выходе из воды не пересекаются в действительности. Однако луч 2, продолженный обратно в толщу воды, пересекает луч 1 , шедший перпендикулярно поверхности раздела вода-воздух, в точке $\mathrm{P}^{\prime}$. Очевидно, что мы получили видимое положение точки дна водоема Р. Таким образом, условия и вопрос задачи можно сформулировать в наших обозначениях: кажущаяся глубина водоема $\mathrm{AP}^{\prime}=3$ м, искомая глубина $\mathrm{AP}$.

Лучи 1 и 2 образуют малый угол $\beta$, поскольку при формировании изображения дна водоема оба луча должны попадать в глаз человека, проходя в небольшое по размеру отверстие зрачка, и фокусироваться хрусталиком на сетчатке. Поэтому параксиальное приближение в данной задаче вполне обоснованно. 
Согласно закону преломления В. Снеллиуса: $\mathrm{n}_{1} \cdot \sin \alpha=\mathrm{n}_{2} \cdot \sin \beta$. В условиях нашей задачи $\mathrm{n}_{1}=1$ (воздух); $\mathrm{n}_{2}=4 / 3$ (вода). Применим параксиальное приближение:

$$
\begin{cases}\alpha \rightarrow 0 & \sin \alpha \approx \operatorname{tg} \alpha \\ \beta \rightarrow 0 & \sin \beta \approx \operatorname{tg} \beta\end{cases}
$$

Тогда, учитывая определение тангенсов углов падения и преломления, получим:

$$
\sin \alpha=\operatorname{tg} \alpha=\frac{\mathrm{AB}}{\mathrm{AP}^{\prime}} ; \sin \beta=\operatorname{tg} \beta=\frac{\mathrm{AB}}{\mathrm{AP}} .
$$

Подставим полученные выражения синусов в закон Снеллиуса:

$$
\mathrm{n}_{1} \cdot \frac{\mathrm{AB}}{\mathrm{AP}^{\prime}}=\mathrm{n}_{2} \cdot \frac{\mathrm{AB}}{\mathrm{AP}} \Rightarrow \mathrm{AP}=\mathrm{AP}^{\prime} \cdot \frac{\mathrm{n}_{2}}{\mathrm{n}_{1}}=3 \cdot \frac{4 / 3}{1}=4 \mathrm{M} \text {. }
$$

Таким образом, истинная глубина водоема в $4 / 3$ раза больше видимой.

Задача 3. Улитка размера а сидит на дальней стенке прямоугольного аквариума ширины $\ell$. В сколько раз изменится видимый угловой размер улитки, если из аквариума слить воду? Наблюдатель расположился на расстоянии L от аквариума.[2, с. 309]

Решение.

Будем считать, что стенки аквариума тонкие, а потому они не вносят искажение в ход лучей, будто световые лучи сразу выходят из воды в воздух, «не замечая» стеклянных стенок. Будем считать также, что размер улитки гораздо меньше, чем расстояние от нее до наблюдателя. Тогда видимый угловой размер (в радианах) для случая аквариума без воды можно найти по формуле длины дуги окружности:

$$
\alpha=\frac{\mathrm{a}}{\mathrm{L}+\ell}
$$

Так как слой воды приближает к нам улитку в $\mathrm{n}=4 / 3$ (показатель преломления воды) раз, то видимый угловой размер улитки в случае, когда в аквариуме есть вода можно определить по формуле:

$$
\alpha_{0}=\frac{\mathrm{a}}{\mathrm{L}+\ell / \mathrm{n}} \text {. }
$$

Тогда уменьшение видимого углового размера можно определить отношением:

$$
\frac{\alpha_{0}}{\alpha}=\frac{\mathrm{L}+\ell}{\mathrm{L}+\ell / \mathrm{n}}=\frac{\mathrm{L}+\ell}{\mathrm{n} \cdot \mathrm{L}+\ell} \cdot \mathrm{n}
$$

Задача 4. Светящаяся точка находится в воздухе на расстоянии $\mathrm{AP}=0.6$ м от выпуклой поверхности стекла радиусом $\mathrm{R}=7.5 \mathrm{~cm}$. Определите расстояние между изображениями точки, которые образуются лучами, отраженными и преломленными поверхностью. Коэффициент преломления стекла $\mathrm{n}=1.5$. $[3$, c. 386] 


\section{Решение.}

Для построения изображения светящейся точки $\mathrm{A}$, получаемого при отражении от сферической поверхности, используем дополнительное построение. Проведем вспомогательный луч через центр сферы О параллельно падающему на поверхность лучу АМ.

Вспомогательный луч пересекает фокальную плоскость в точке побочного фокуса $\mathrm{F}^{\prime}$, в котором собираются все продолжения лучей параллельных вспомогательному лучу, поэтому отраженный луч будет проходить через точки $\mathrm{F}^{\prime}$ и М. Продолжение отраженного от точки М сферы луча пересекается с главной оптической осью в точке $\mathrm{A}^{\prime}$ - изображении точки $\mathrm{A}$.

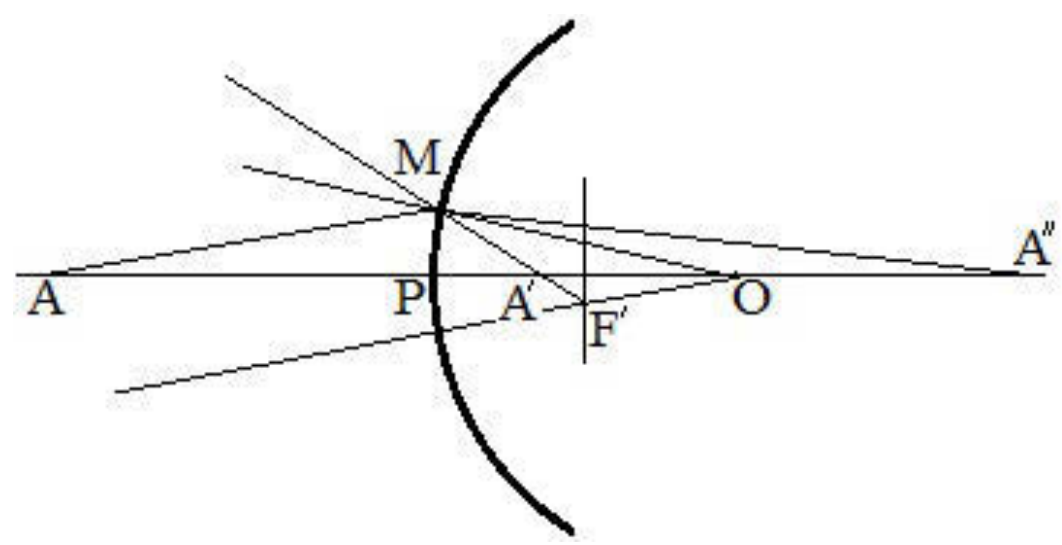

Рис. 5. Получение двух изображений точки на стеклянной сферической поверхности

Очевидно, что положение точки $\mathrm{A}^{\prime}$ на главной оптической оси будет определяться выражением формулы сферического зеркала, полученного из параксиального приближения ранее. С учетом выпуклости зеркала и правила знаков можно получить:

$$
\begin{gathered}
\frac{1}{\mathrm{AP}}-\frac{1}{\mathrm{PA}^{\prime}}=-\frac{2}{\mathrm{R}} ; \\
\mathrm{PA}^{\prime}=\frac{\mathrm{R} \cdot \mathrm{AP}}{2 \mathrm{AP}+\mathrm{R}}=\frac{7.5 \cdot 60}{2 \cdot 60+7.5}=3.53(\mathrm{~cm}) .
\end{gathered}
$$

Для нахождения положения изображения точки, получаемого в результате преломления луча на сферической поверхности раздела двух сред, воспользуемся формулой преломления на сферической поверхности, выведенной ранее из параксиального приближения. С учетом наших обозначений получим:

$$
\begin{gathered}
\frac{1}{\mathrm{AP}}+\frac{\mathrm{n}}{\mathrm{PA}^{\prime \prime}}=\frac{\mathrm{n}-1}{\mathrm{R}} ; \\
\mathrm{PA}^{\prime \prime}=\frac{\mathrm{n} \cdot \mathrm{R} \cdot \mathrm{AP}}{(\mathrm{n}-1) \mathrm{AP}-\mathrm{R}}=\frac{1.5 \cdot 7.5 \cdot 60}{0.5 \cdot 60-7.5}=30(\mathrm{~cm}) .
\end{gathered}
$$

Тогда расстояние между двумя изображениями:

$$
\mathrm{A}^{\prime} \mathrm{A}^{\prime \prime}=\mathrm{PA}^{\prime}-\mathrm{PA}^{\prime \prime}=30-3.53=26.5(\mathrm{~cm}) \text {. }
$$


Задача 5. На дне аквариума, имеющего форму шара радиусом $\mathrm{R}$, находится рыбка. Где увидит рыбку наблюдатель, смотрящий на нее сверху, если показатель преломления воды равен $\mathrm{n}$ ? Стенки аквариума очень тонкие. [3, с. 386]

Решение.

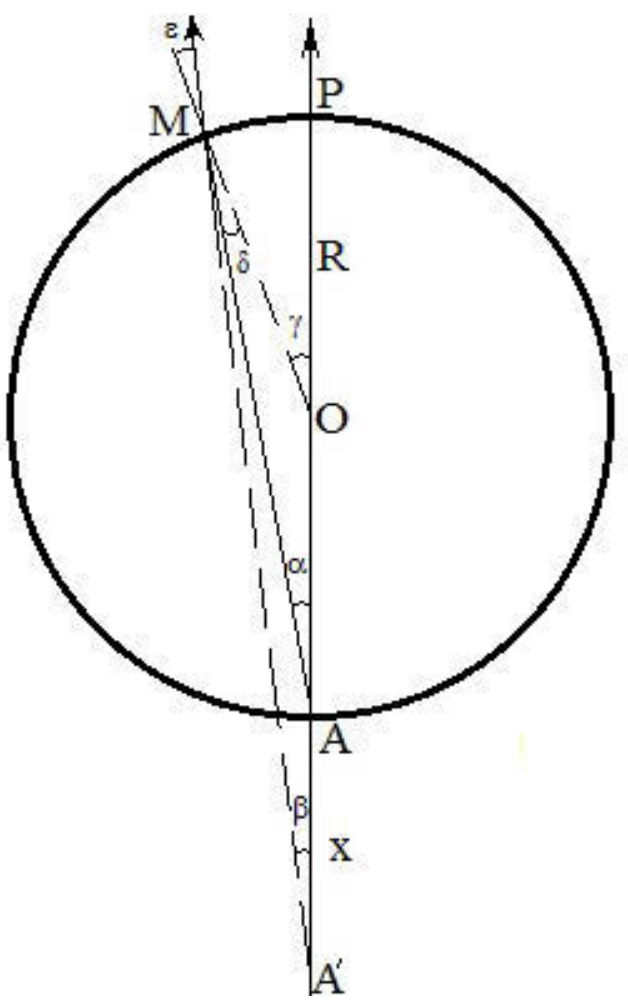

Рис. 6. Рыбка в аквариуме

Условия данной задачи подразумевают использование параксиального приближения, поскольку наблюдение рыбки А производится невооруженным глазом человека и для такой ситуации будут справедливы все те же замечания, которые были сделаны в задаче № 2 .

Угол $\gamma$ - смежный для треугольников:

$\Delta \mathrm{AMO}$, значит, по теореме о смежных углах имеем $\gamma=\alpha+\delta$;

$\Delta \mathrm{A}^{\prime} \mathrm{MO}$, поэтому $\gamma=\beta+\varepsilon$.

Запишем закон Снеллиуса в параксиальном приближении: $\varepsilon=\mathrm{n} \cdot \delta$. Преобразуем все три уравнения в систему:

$$
\left\{\begin{array}{l}
\varepsilon=\gamma-\beta \\
n \cdot \delta=n \cdot \gamma-n \cdot \alpha \\
\varepsilon=n \cdot \delta
\end{array} \mid \Rightarrow \gamma-\beta=n \cdot \gamma-n \cdot \alpha \Rightarrow n \cdot \alpha-\beta=(n-1) \cdot \gamma\right.
$$

Введем обозначения:

$\mathrm{AP}=2 \mathrm{R} ; \mathrm{OP}=\mathrm{R} ; \mathrm{A}^{\prime} \mathrm{P}=2 \mathrm{R}+\mathrm{x}$, где $\mathrm{x}-$ искомое расстояние от дна аквариума до изображения рыбки. Обозначенные ранее углы можно найти по формуле длины дуги МР : 


$$
\alpha=\frac{\mathrm{MP}}{\mathrm{AP}}=\frac{\mathrm{MP}}{2 \mathrm{R}} ; \beta=\frac{\mathrm{MP}}{\mathrm{A}^{\prime} \mathrm{P}}=\frac{\mathrm{MP}}{2 \mathrm{R}+\mathrm{x}} ; \gamma=\frac{\mathrm{MP}}{\mathrm{OP}}=\frac{\mathrm{MP}}{\mathrm{R}} .
$$

Тогда, подставляя последние выражения в формулу, полученную из системы, найдем х :

$$
\begin{gathered}
\frac{n \cdot M P}{2 R}-\frac{M P}{2 R+x}=\frac{(n-1) M P}{R} ; \\
\frac{1}{2 R+x}=\frac{n-2 n+2}{2 R}=\frac{2-n}{2 R} ; \\
(2 R+x)(2-n)=2 R ; \\
x(2-n)=2 R(n-1) ; \\
x=\frac{2 R(n-1)}{2-n}=\frac{2 R(4 / 3-1)}{2-4 / 3}=R
\end{gathered} .
$$

Таким образом, изображение рыбки $\mathrm{A}^{\prime}$ находится от нее на расстоянии равном радиусу.

Приведенная выше цепочка задач была апробирована на развивающих занятиях с учащимися школ и в процессе тренинга участников Всероссийской олимпиады школьников по физике.

Подведем итоги: параксиальное приближение действительно помогает решать олимпиадные задачи геометрической оптики, позволяя обойти математические сложности при решении тригонометрических уравнений, приводя их к обычным линейным уравнениям.

\section{Список литературы:}

1. Ландсберг Г.С. Оптика. М.: Наука. 1976. 928 с.

2. Воробьев И.И., Зубков П.И., Савченко О.Я. и др. Задачи по физике. М.: Наука. 1988. 416 с.

3. Балаш В.А. Задачи по физике и методы их решения. М.: Просвещение, 1974. 430 с.

(C) 2014, Сиделов Д.И.

О необходимости применения параксиального приближения при решении олимпиадных задач геометрической оптики
(C) 2014, Sidelov D.I.

The need for the use of the paraxial approximation in solving olympiad problems of geometrical optics 